\title{
BMJ Open Impact of parents' education on variation in hospital admissions for children: a population-based cohort study
}

Frank Olsen (D) , ${ }^{1,2}$ Lise Balteskard, ${ }^{2}$ Bård Uleberg, ${ }^{1,2}$ Bjarne K Jacobsen, ${ }^{1,2,3}$ Ivar Heuch, ${ }^{4}$ Atle Moen ${ }^{5}$

To cite: Olsen F, Balteskard L, Uleberg B, et al. Impact of parents' education on variation in hospital admissions for children: a population-based cohort study. BMJ Open 2021;11:e046656. doi:10.1136/ bmjopen-2020-046656

- Prepublication history and additional supplemental material for this paper are available online. To view these files, please visit the journal online (http://dx.doi.org/10.1136/ bmjopen-2020-046656).

Received 05 November 2020 Accepted 23 May 2021

Check for updates

(C) Author(s) (or their employer(s)) 2021. Re-use permitted under CC BY-NC. No commercial re-use. See rights and permissions. Published by BMJ.

For numbered affiliations see end of article.

Correspondence to

Frank Olsen;

frank.olsen@helse-nord.no

\section{ABSTRACT}

Objectives To assess the impact of parental educational level on hospital admissions for children, and to evaluate whether differences in parents' educational level can explain geographic variation in admission rates.

Design National cohort study.

Setting The 18 hospital referral areas for children in Norway.

Participants All Norwegian children aged 1-16 years in the period 2008-2016 and their parents.

Main outcome measures Age- and gender-adjusted admission rates and probability of admission.

Results Of 1538189 children, 156087 (10.2\%) had at least one admission in the study period. There was a nearly twofold (1.9) variation in admission rates between the hospital referral areas (3113 per 100000 children, $95 \%$ Cl: 3056 to 3169 vs $1627,95 \%$ Cl: 1599 to 1654). Area level variances in multilevel analysis did not change after adjusting for parental level of education. Children of parents with low level of education (maternal level of education, low vs high) had the highest admission rates (2016: $2587,95 \% \mathrm{Cl}$ : 2512 to 2662 vs $1810,95 \% \mathrm{Cl}$ : 1770 to 1849), the highest probability of being admitted (OR: $1.18,95 \% \mathrm{Cl}: 1.16$ to 1.20 ), the highest number of admissions (incidencerate ratio: $1.05,95 \% \mathrm{Cl}: 1.01$ to $1.10)$ and admissions with lower cost $(-0.5 \%, 95 \% \mathrm{Cl}$ : $-1.2 \%$ to $0.3 \%$ ).

Conclusions Substantial geographic variation in hospital admission rates for children was found, but was not explained by parental educational level. Children of parents with low educational level had the highest admission probability, and the highest number of admissions, but the lowest cost of admissions. Our results suggest that the variation between the educational groups is not due to differences in medical needs, and may be characterised as unwarranted. However, the manner in which health professionals communicate and interact with parents with different educational levels might play an important role.

\section{INTRODUCTION}

Studies on geographic variation in healthcare utilisation started with Glover in 1938, who found large geographic variation in rates of tonsillectomy among English school children. In 1973, Wennberg and Gittelsohn
Strengths and limitations of this study

- A complete national population cohort of children, both admitted and non-admitted, and their parents was studied, eliminating selection bias.

- Individualised time-dependent data eliminate measurement errors and ecological fallacies.

- A study period of 9 years ensures robust results.

- Information about the parents' income and occupational status was not available for this study.

- Reliable prevalence data at the population level on the morbidity in childhood are unavailable in Norway.

published similar findings in the USA. ${ }^{2}$ Geographic variation in healthcare utilisation for children and adults has later been described independently of how healthcare delivery is organised..$^{3-5}$

While variation has primarily been studied in the context of geographic differences, it also exists related to differences in socioeconomic status (SES). SES is the social standing or class of an individual or a group, and is often measured as a combination of education, income and occupation. If variation cannot be explained by differences in patient needs or patient preferences, it may be considered unwarranted. ${ }^{6}$ An inverse association between SES and hospitalisation for children has been documented. ${ }^{78}$ Variation between SES groups may reflect differences in disease prevalence or needs due to lifestyle or environmental factors, but may also be related to other factors, such as different doctor-patient communication. ${ }^{9-11}$

Norway provides free access to healthcare independent of income and SES within a single-payer publicly owned healthcare system. The health of Norwegian children is excellent with an under-five child mortality rate of 2.5 per 1000 live births in 2018 compared with 6.5 in the USA. ${ }^{12}$ Nonetheless, 
the Norwegian Child Healthcare Atlas published in $2015^{5}$ and the Neonatal Healthcare Atlas published in $2016^{13}$ found relatively large geographic variation between hospital referral areas in admission rates and rates of treatment procedures. This variation could not be explained by differences in morbidity or patient preferences. Higher admission rates are not necessarily associated with better outcomes ${ }^{14}$ and may expose patients to risk of complications from treatment or hospital-acquired infections. In general, children should only be admitted to hospital if outpatient care cannot be provided with an equal or better outcome.

Using national registers, the paediatric cohort of children aged 1-16 years in Norway over a 9-year period was matched with parental educational attainment. This is the first study with individual data on a complete national cohort of children, both hospitalised and nonhospitalised, and their parents' educational level. Parental educational level was used as measure of SES.

The aim of this population-based study was to describe geographic variation and explore the effect of parental educational level on hospital admissions for children. We address the following questions: Can geographic variation in admission rates for children between hospital referral areas be explained by parental educational level? What are the impacts of parental educational level on whether a child is admitted to hospital or not? If a child is admitted, does parental educational level impact the number of admissions, disease severity and cost of admissions?

\section{MATERIALS AND METHODS}

\section{Study design and data sources}

The study population was defined using combined data from the Norwegian Patient Register (NPR) and Statistics Norway (SSB) and included a complete cohort of all Norwegian children aged 1-16 years from 1 January 2008 to 31 December 2016. Data were linked by an encrypted serial number derived from the unique 11-digit personal identifier held by all persons living in Norway. The data from SSB consisted of parental level of education each year, number and birth year of siblings, year of birth of the parents, gender and year of birth and residential municipality. The data from NPR consisted of patient demographics (residential information, age and gender), start and end date for the visit, name of hospital, type of visit, diagnoses and procedures performed. In Norway, all hospitals submit data to NPR for registration and reimbursement purposes.

\section{Definitions}

Hospital admissions for medical diagnoses (non-surgical diagnosis-related group (DRG) grouping) of at least 1 day were included in the analysis. In addition, admissions with certain primary diagnoses not considered paediatric medicine were excluded (for a detailed list of diagnoses, see online supplemental file 1). Admission episodes with less than 8 hours in between department stays were considered as one admission. Admissions that consisted of two or more department stays were registered as medical visits if all stays were registered with a medical diagnosis. Admissions were registered by the year of discharge. In addition, four subsamples of admissions were defined using primary and secondary diagnosis codes: gastroenteritis, viral and bacterial infections (excluding gastroenteritis), epilepsy and asthma (for details, see online supplemental file 1).

Parental educational level was coded using the international standard classification of education. Larger numbers represented higher educational level; 0 indicated less than primary education and 8 indicated a doctorate or equivalent, while 9 was not classified and regarded as missing. Educational level was recoded into three categories: low (0-2), medium (3-5) and high (6-8). The number of siblings was computed each year according to birth year, and analysed as a dichotomous variable; only child or child with siblings.

\section{Statistical analyses}

Data were analysed using SAS V.9.4 (SAS Institute, Cary, North Carolina, USA). The data were structured as one record per child per year, and the variables were time-dependent.

Age- and gender-adjusted admission rates were calculated for children with medical admissions in the hospital referral areas corresponding to the geographic areas served by the 18 Norwegian hospital trusts. The direct method of standardisation was applied, with three age groups (1-3, 4-9 and 10-16 years). Both annual and overall rates for the period 2008-2016 were calculated separately for parents' educational level categories. The reference population was the annual average of all children aged 1-16 years in Norway in the period.

Independent variables included were child's age and gender, maternal age, maternal and paternal level of education (categorical) and being an only child or not. Due to the high correlation between parents' ages, father's age was not included in the analysis. Restricted cubic splines ( 4 knots) for age with interaction terms for gender were applied, to adjust for child's age and gender. High level of education and only child were set as reference categories. In any particular analysis, observations with relevant missing data were excluded.

Admission was a dichotomous variable for each child, and the year of the first admission was used as admission time point. For children with multiple admissions, only the year of the first admission was considered. Admission was analysed using discrete-time survival analysis (based on binary logistic regression). ${ }^{15}$

In the analysis of the number of admissions, and the cost or severity of the admission, the study population was restricted to children with admissions only, and the independent variables were defined by the year of the first admission. The number of admissions was counted for each child in the year of the first admission. As the number of admissions is a counter variable with values 


\begin{tabular}{lll}
\hline $\begin{array}{l}\text { Table } 1 \text { Characteristics of children (1-16 years) } \\
\text { 2008-2016 }\end{array}$ & & \\
\hline Number of children (\% admitted) & 1538189 & $(10.15)$ \\
\hline Child's age, mean (SD) & 6.16 & $(5.19)$ \\
\hline Boys, $\mathrm{n}(\%)$ & 789635 & $(51.34)$ \\
\hline Mother's age, mean (SD) & 35.81 & $(6.83)$ \\
\hline Missing, $\mathrm{n}(\%)$ & 7643 & $(0.50)$ \\
\hline Father's age, mean (SD) & 38.87 & $(7.59)$ \\
\hline Missing, $\mathrm{n}(\%)$ & 39457 & $(2.57)$ \\
\hline Mother's education ISCED, mean (SD) & 4.7 & $(1.73)$ \\
\hline Father's education ISCED, mean (SD) & 4.48 & $(1.70)$ \\
\hline Mother's educational level, categorical & & \\
\hline Low, $\mathrm{n}(\%)$ & 261226 & $(16.98)$ \\
\hline Medium, $\mathrm{n}(\%)$ & 488739 & $(31.77)$ \\
\hline High, $\mathrm{n}(\%)$ & 703200 & $(45.72)$ \\
\hline Missing, $\mathrm{n}(\%)$ & 85024 & $(5.53)$ \\
\hline Father's educational level, categorical & & \\
\hline Low, $\mathrm{n}(\%)$ & 267667 & $(17.40)$ \\
\hline Medium, $\mathrm{n}(\%)$ & 644727 & $(41.91)$ \\
\hline High, $\mathrm{n}(\%)$ & 515724 & $(33.53)$ \\
\hline Missing, $\mathrm{n}(\%)$ & 110071 & $(7.16)$ \\
\hline No of siblings, mean (SD) & 1.53 & $(1.27)$ \\
\hline Missing, $\mathrm{n}(\%)$ & 4697 & $(0.31)$ \\
\hline Only child, $\mathrm{n}$ (\%) & 282498 & $(18.37)$ \\
\hline
\end{tabular}

*Based on the start of the follow-up for each child.

ISCED, international standard of classification of education.

greater or equal to 1 , truncated negative binomial regression was applied. DRG-weight of the first admission was used as a measure of cost and disease severity. DRG-weight was analysed with linear regression. DRG-weight was highly right-skewed and was therefore log-transformed. Also, the sum of DRG-weights in the first year with admission and sum of all DRG-weights throughout the period were calculated and analysed.

To control for the impact of parental level of education on geographic variation, we conducted sensitivity analyses, that is, multilevel analysis with random intercept for the hospital referral areas. This was done for the survival analysis of admission and DRG-weight. The analyses were stratified by gender and performed with restricted cubic splines ( 4 knots) for age. The full model with all the independent variables was compared with a reduced model without parental education.

\section{RESULTS}

A total of 1538189 children were included in the analysis with a total of 8946984 entries over the study period (2008-2016). Of these, 156087 (10.2\%) children had at least one admission (table 1). There were 198293 admissions during the year of the first admission, with
Rates per 100000 children

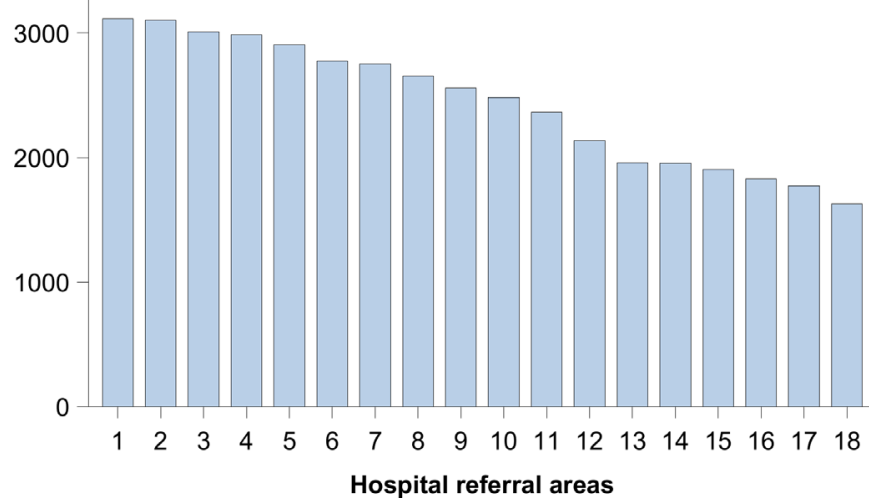

Figure 1 Age- and gender-adjusted hospital admission rates per 100000 children, by hospital referral areas, average total rates.

an average of 1.27 (SD: 1.12) admissions per child. The mean DRG-weight for the first admission was 0.76 (SD: $0.59)$.

There was a near twofold (1.9) difference in admission rates between the hospital referral areas (area 1: 3113 per 100000 children, $95 \%$ CI: 3056 to 3169 vs area 18: 1627, 95\% CI: 1599 to 1654 ) (figure 1 and online supplemental table S1). Admission rates increased as the level of education for both the mother and father decreased. The effect was consistent with a slight decrease in overall admission rates over time (figure 2 and online supplemental table S1). Children of mothers with low level of education had on average $36 \%$ higher admission rates compared with children of mothers with high level of education (in 2016: $2587,95 \%$ CI: 2512 to 2662 vs $1810,95 \%$ CI: 1770 to 1849). The same pattern was found in all hospital referral areas independent of total admission rates in each area (figure 3 and online supplemental table S1). The results from the analyses of the subsamples of admissions were similar (online supplemental figures S1-S3).

In the analyses adjusted for other factors, the probability of admission increased with decreasing maternal

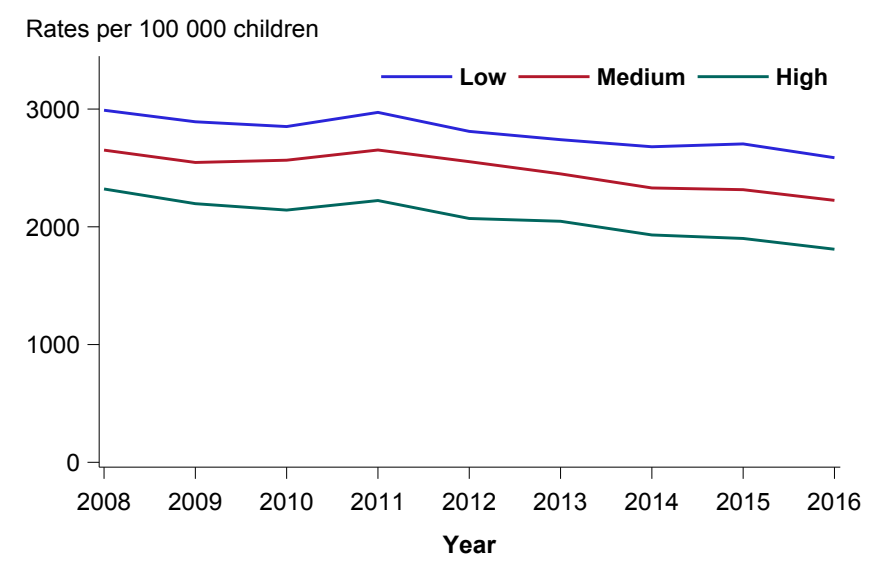

Figure 2 Age- and gender-adjusted hospital admission rates per 100000 children, annual rates, by mothers' educational level. 


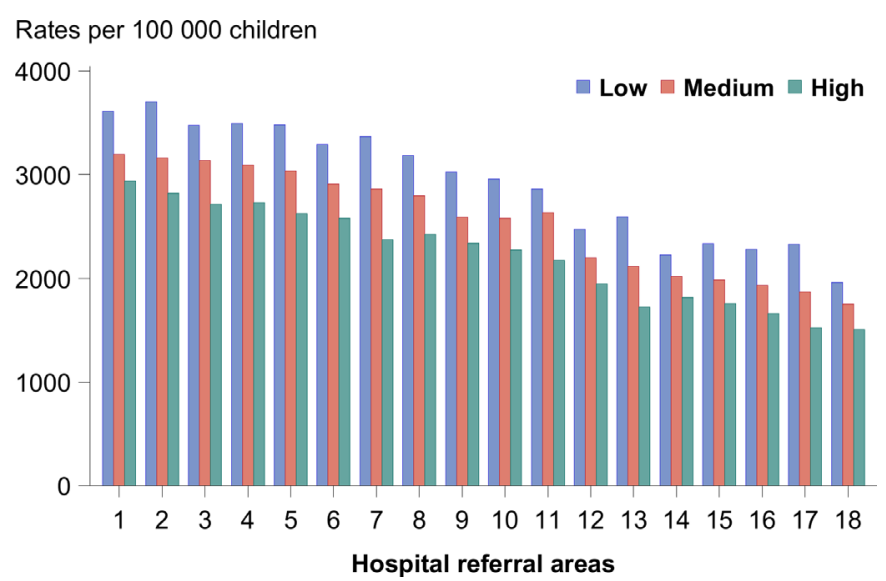

Figure 3 Age- and gender-adjusted hospital admission rates per 100000 children, average rates, by hospital referral areas (sorted in the same order as figure 1) and mothers' educational level.

and paternal level of education (low vs high maternal level of education-OR: $1.18,95 \%$ CI: 1.16 to 1.20 ; low vs high paternal level of education-OR: 1.21, 95\% CI: 1.19 to 1.23) (table 2). The probability of admission decreased with increased maternal age (per 5years-OR: 0.94, 95\% CI: 0.93 to 0.94 ) and being an only child (OR: 0.91 , $95 \%$ CI: 0.90 to 0.93 ). Results from multilevel analysis were similar (table 3). Multilevel analysis without parental level of education (the reduced model in table 3) resulted in similar area-level variance, indicating that differences in parental level of education do not explain the geographic variation and vice versa. Analysis stratified by children's age also found a negative parental educational gradient for almost all ages. In addition, in the analyses of the subsamples the negative educational gradient was found (online supplemental table S2).
Children of parents with low or medium level of education had a higher number of admissions than children of parents with a high level of education (incidence rate ratios (IRRs): $1.05,95 \%$ CI: 1.01 to 1.10 ), low vs high paternal level of education (IRR: 1.05, 95\% CI: 1.01 to 1.09) (table 2). The number of admissions per child increased with maternal age (per 5years-IRR: 1.03, 95\% CI: 1.02 to 1.05$)$.

DRG-weight was highest for children of parents with high level of education. The differences from the reference category were $<2 \%$ and mostly statistically significant (low vs high maternal level of education $(-0.5 \%, 95 \%$ CI: $-1.2 \%$ to $0.3 \%$ ), low vs high paternal level of education $(-1.9 \%, 95 \%$ CI: $-2.7 \%$ to $-1.1 \%))$. DRG-weight increased with maternal age (per 5 years-1.2\%, 95\% CI: $0.9 \%$ to $1.4 \%$ ), while being an only child was associated with a lower DRG-weight $(-1.1 \%, 95 \%$ CI: $-1.8 \%$ to $-0.3 \%)$ (table 2). Applying the two alternative measures for cost also resulted in the highest sums of DRG-weights for children of parents with high level of education. The results from the multilevel analysis were similar (table 3 ).

\section{DISCUSSION}

\section{Principal findings}

Children of parents with low level of education had the highest admission rates, while children of parents with high level of education had the lowest admission rates. This was consistent both over time and across hospital referral areas. The geographic variation in admission rates was nearly twofold but was not explained by differences in parents' level of education. The analyses based on individual data from all Norwegian children aged 1-16 during 2008-2016 (table 2) supported the results from the ecological analyses of admission rates (figure 3 ).

\begin{tabular}{|c|c|c|c|c|}
\hline Covariate & Category & Admission (OR)* & Number of admissions (IRR)† & DRG-weight (\%)‡ \\
\hline Mother's age & Per 5 years & 0.94 (0.93 to 0.94$)$ & 1.03 (1.02 to 1.04$)$ & $1.2(0.9$ to 1.4$)$ \\
\hline \multirow{3}{*}{$\begin{array}{l}\text { Mother's } \\
\text { educational level }\end{array}$} & Low & $1.18(1.16$ to 1.20$)$ & 1.05 (1.01 to 1.10$)$ & $-0.5(-1.2$ to 0.3$)$ \\
\hline & Medium & 1.11 (1.09 to 1.12$)$ & 1.04 (1.01 to 1.07$)$ & $-1.5(-2.1$ to -0.9$)$ \\
\hline & High & 1.0 & 1.0 & 0 \\
\hline \multirow{3}{*}{$\begin{array}{l}\text { Father's educational } \\
\text { level }\end{array}$} & Low & 1.21 (1.19 to 1.23$)$ & 1.05 (1.01 to 1.09$)$ & $-1.9(-2.7$ to -1.1$)$ \\
\hline & Medium & 1.14 (1.13 to 1.16$)$ & 1.04 (1.01 to 1.08$)$ & $-1.7(-2.4$ to -1.1$)$ \\
\hline & High & 1.0 & 1.0 & 0 \\
\hline \multirow[t]{2}{*}{ Only child } & Yes & 0.91 (0.90 to 0.93 ) & 0.99 (0.95 to 1.02$)$ & $-1.1(-1.8$ to -0.3$)$ \\
\hline & No & 1.0 & 1.0 & 0 \\
\hline
\end{tabular}

*Survival analysis with binary logistic regression, restricted cubic splines knots for age $(4,7,10,13), n=7$ 701 336. Adjusted effects are ORs. †Truncated negative binomial regression, restricted cubic splines knots for age $(1,3,6,12), n=143697$. Adjusted effects are IRRs. łLinear regression with log-transformed outcome, restricted cubic splines knots for age $(1,3,7,12), n=143664$. Adjusted effects are percentage differences from the reference category (100x(exp(estimate)-1)).

DRG, diagnosis-related group; IRRs, incidence rate ratios. 
Table 3 Results from multilevel analysis for admission and DRG-weight, with random intercept for hospital referral area and restricted cubic splines for age, stratified by gender. Point estimates with $95 \% \mathrm{Cls}$

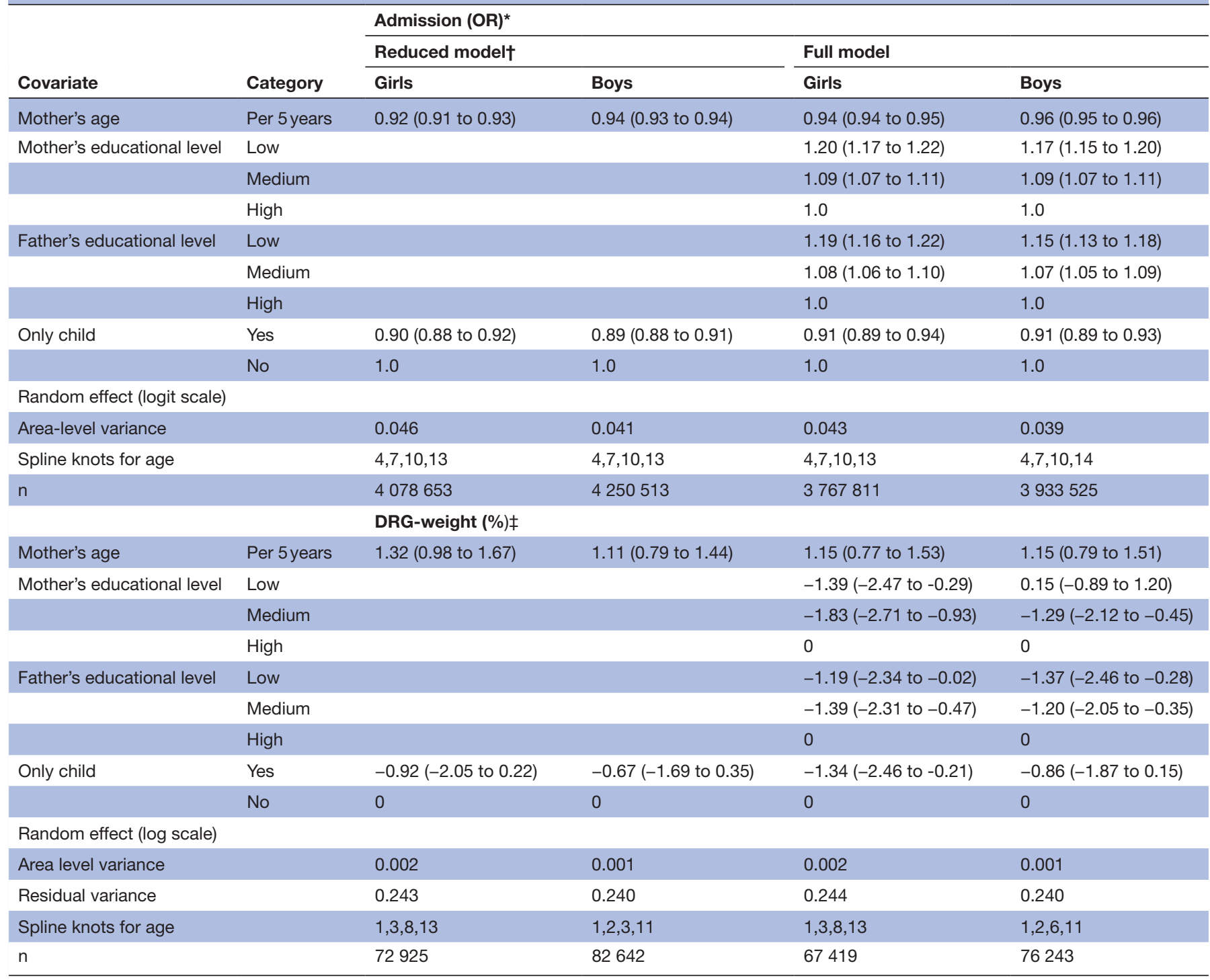

*Survival analysis with binary logistic regression. Adjusted effects are ORs.

†Reduced model: without parental education.

łLinear regression with log-transformed outcome. Adjusted effects are percentage differences from the reference category (100×(exp(estimate)-1)).

DRG, diagnosis-related group.

They further indicated that children of parents with low and medium level of education also had somewhat more frequent admissions per child, while the cost or severity per admission was slightly lower for these children compared with children of parents with high level of education.

\section{Comparison with previous studies}

The geographic variation in admission rates is in accordance with the findings of unwarranted variation reported in the Child healthcare atlas for Norway. ${ }^{5}$ Our present study found the same variation over a time span of 9 years as the 5-year duration in the atlas. The observed geographic variation in the atlas and in this study can mainly be attributed to two different mechanisms for unwarranted variation, preference-sensitive and supply-sensitive care. ${ }^{6}$
Preference-sensitive care represents practice, preferences and beliefs of a single clinician or department rather than a clear evidence-based approach and unwarranted variation is caused by differences in clinical practice or patients' participation in care decisions. Supply-sensitive care refers to medical services for which utilisation rates are sensitive to local availability of healthcare resources, and unwarranted variation is due to differences in capacity. It is reasonable to assume that the observed variation between the hospital referral areas is related to both differences in clinical practice and differences in capacity. The inverse gradient between admission rates and parental level of education is in accordance with findings by others. ${ }^{8} 1617$ Similar results have also been found for adults in systems with universal healthcare coverage. ${ }^{18}$ 
DRG-weight may serve as a crude indicator of disease severity. DRG-weight was positively associated with increasing level of parental education, suggesting that the conditions causing the admission were slightly less severe among children of parents with low level of education. Previous studies have found higher treatment costs for children with low SES. ${ }^{7819}$ Nonetheless, the number of admissions was about 5\% higher among children of parents with low level of education compared with children of parents with high level of education in our study. Moreover, the sum of DRG-weights in the first year with admission and the sum of all DRG-weights throughout the period for children of parents with high level of education were slightly higher than that of children of parents with low and medium level of education. The contrast with previous studies may be related to their use of ecological SES measures or SES fixed to a point in time not necessarily corresponding to the hospital admission. Unlike these studies, we used individualised paired data for each child-parent couple at the year of admission.

\section{Possible explanations of our findings}

Out-of-pocket payment or lack of health insurance may be an obstacle to disadvantaged groups seeking healthcare. ${ }^{20}$ All healthcare for children under the age of 16 in Norway is free of charge, and parents are economically compensated for the loss of income if admitted to hospital with their child. This most likely excludes a significant effect of economic restraints on access to healthcare for children.

Thus, there must be other factors involved explaining the variation associated with education. First, there may be differences in disease prevalence and medical needs or informed preferences related to parents' level of education.

There is increasing evidence of a positive relationship between SES and health outcomes throughout the life span. ${ }^{21}$ However, most SES factors influencing health status are related to exposure over time, during a critical period or through the pathway of learnt lifestyle. As a consequence, the major impact of SES on health becomes apparent later on in life, not during childhood. ${ }^{22}$

There is a paucity of reliable population-based disease prevalence data in children. Although utilisation of healthcare resources is commonly interpreted as an indicator of prevalence, this is hardly correct given the large geographic variation found by us and others. ${ }^{3-5}$ Disease prevalence is not consistently higher in children with low SES. Atopic disease and allergies occur more frequently among children with high SES. ${ }^{23-25}$ The prevalence of asthma did not show an association with SES, while severe asthma was most prevalent in low SES groups according to an analysis by Mielck et al. ${ }^{26} \mathrm{~A}$ recent Danish study found a significantly higher risk of childhood nervous system tumours of all types among children with highly educated parents or mothers with high income. ${ }^{27}$ A German crosssectional study concluded that only a few health indicators such as obesity occurred more frequently in socially disadvantaged children. ${ }^{25}$ The pattern of admission rates found in our study does not necessarily fit with the heterogeneous pattern of SES-related prevalence for diseases in childhood, and care should be taken not to interpret admission rates as a reflection of prevalence.

The majority of paediatric hospital admissions in Norway are related to acute and less-severe disease, and most children admitted are only hospitalised once or twice during childhood. The standard of living in Norway is high, income inequality is relatively small and few children live in poverty. It may therefore be questioned if variation in admission rates as large as $36 \%$ between educational groups is reasonable and if it is solely related to differences in disease prevalence.

Alternatively, the variation associated with education and SES may be related to other factors than the child's health status. Both differences in preferences and capacity may contribute to large variation in healthcare usage between geographic areas. These mechanisms are usually unintended and not recognised by providers. It may be due to attitudes or beliefs held by either parents or physicians, which may impact the decision of admission.

Finnvold found that despite the strict practice of admission criteria, children with severe asthma are more likely to be admitted to a specialised asthma hospital if their parents have higher education, participate in patient organisations or there is a physician in the family. ${ }^{28}$ This indicates that manoeuvrability related to higher education and social capital increases the chances of admission to a specialised hospital. One of the mechanisms underlying SES differences in healthcare usage may be found in the concept of health literacy, which captures the difficulties parents may encounter in finding their way through the healthcare system. ${ }^{29}$ Health literate parents may be more capable of understanding and discussing treatment options on equal grounds with their physician and therefore avoid admissions with little benefit over outpatient care. The association between educational level and health literacy is well documented. ${ }^{30}$ It is demonstrated that low functional health literacy is associated with suboptimal use of healthcare services. ${ }^{31}$

It has been claimed that parents with higher levels of education or SES are more willing or capable to participate in shared decision-making. ${ }^{932}$ Salvador et alfound an association between parents preferring a passive collaborative role and lower levels of education. ${ }^{33}$ However, parents' willingness to participate may reflect the physician's consulting and communication style. In a metaanalysis on doctor-patient communication related to SES, ${ }^{9}$ physicians gave more information, more explanation, were more emotionally supportive and more often adapted shared decision-making with patients of high SES. Furthermore, patients with low SES received more physical examination. Physicians may presume that patients with low SES are less intelligent, less responsible, less rational and less likely to comply with medical advice. ${ }^{10}$ This may affect decisions on whether to admit the child to inpatient care or not. ${ }^{11}$ Therefore, the physician may have a lower threshold for admitting children 
from families with low and medium SES. This is also in accordance with our findings that there is a higher probability for admission and lower cost for children of parents with low level of education. Such decisions may not be rational and fact-based, but rather reflect unrecognised assumptions about people with a different background and SES than the physicians.

If the variation in admission rates between educational groups does not reflect needs or informed preferences, the variation may be characterised as unwarranted. The correct rate reflects the decision of fully informed patients and families, while variation in rates reflects both local practice and the influence of physicians on parental decisions. ${ }^{34}$ If the extent of shared decision-making increases with parental SES, the admission rate of children with higher SES may better reflect actual needs based on medical criteria and preferences.

\section{Strengths and limitations}

The strength of this study is the use of individualised yearly matched data for each child and parents' level of education, for both admitted and non-admitted children. The parental level of education in Norway increased during the study period, with a 5 percentage points increase in the proportion with high level of education. In the analyses, parental level of education in the year of the child's admission was applied. The study covered a complete national cohort of children over 9 years, with consistent findings both over time and between groups. The completeness of data eliminates selection bias.

A limitation might be that income has not been included as an indicator of SES, which may or may not improve the classification of SES. However, income is volatile and fluctuates considerably over time. Kaarbøe and Carlsen found that for hospital admissions in children under the age of 11 in Norway, the educational gradient dominated the income gradient for SES. ${ }^{7}$ Halldòrsson et al found that education was a more important determinant of healthcare utilisation for children than the financial situation of the families in Nordic countries. ${ }^{16}$

\section{Unanswered questions and future research}

Our data did not allow firm conclusions about a causal relationship, neither between medical needs nor nonmedical factors, and differences in hospital admission rates among children of parents with different educational level. Previous studies on geographic variation in medical care indicated that physician preferences exert a major impact on variation in care. ${ }^{35}$ This may also be true for variation between SES groups, even though the nature and quality of these preferences may be different. More research on how health professionals communicate with patients of different SES and the effects on treatment decisions is needed.

The goal of shared decision-making is to improve the overall quality of clinical decisions, satisfaction and to avoid admissions with no benefit over outpatient care. However, shared decision-making depends on a two-way partnership between the physician and the parents. Parents without sufficient understanding of their child's medical condition are not able to make educated and fully informed decisions. Therefore, tools to improve health literacy among patients/parents and to increase physician's skills in communication are needed.

\section{CONCLUSION}

This population-based cohort study, including all Norwegian children aged 1-16 years, demonstrates that children of parents with low or medium level of education have an increased likelihood of being admitted to inpatient hospital care. Geographic variation in admission rates cannot be explained by differences in parents' level of education. Different admission rates do not necessarily reflect differences in disease prevalence, but may also reflect differences in interaction between the healthcare provider and the child's parents depending on the parents' level of education.

\section{Author affiliations}

${ }^{1}$ Department of Community Medicine, UiT The Arctic University of Norway, Troms $\emptyset$, Norway

${ }^{2}$ Centre for Clinical Documentation and Evaluation (SKDE), Northern Norway Regional Health Authority, Troms $\emptyset$, Norway

${ }^{3}$ Centre for Sami Health Research, Department of Community Medicine, UiT The Arctic University of Norway, Troms $\emptyset$, Norway

${ }^{4}$ Department of Mathematics, University of Bergen, Bergen, Norway

${ }^{5}$ Department of Neonatology, Oslo University Hospital, Oslo, Norway

Contributors FO, LB, BU and AM conceived and designed the study. F0 facilitated and analysed the data. FO, BKJ and IH contributed to the analytical strategy. FO, $\mathrm{LB}$ and $\mathrm{AM}$ drafted the manuscript. All authors participated in the revision of the manuscript.

Funding The publication charges for this article have been funded by a grant from the publication fund of UiT The Arctic University of Norway.

Disclaimer Data from the Norwegian Patient Register have been used in this publication. The interpretation and reporting of these data are the sole responsibility of the authors, and no endorsement by the Norwegian Patient Register is intended nor should be inferred.

Competing interests None declared.

Patient consent for publication Not required.

Ethics approval The study is based on secondary use of clinical administrative data. For this reason, approval from Regional Committees for Medical and Health Research Ethics (REK) is not required, but REK has given exemption from the duty of confidentiality (20627/REK sør-øst A). The project has conducted a Data Protection Impact Assessment (DPIA). Further ethical approval or obtaining informed consent was not required for this study.

Provenance and peer review Not commissioned; externally peer reviewed.

Data availability statement Data may be obtained from a third party and are not publicly available. The original data were not collected by the authors but made available by record-linkage between the Norwegian Patient Registry (NPR) and the Statistics Norway (SSB), using the unique 11 digits personal ID. Individuallevel health data are, by definition, considered to be sensitive information in the Norwegian legislation, even if deidentified and strict confidentiality requirements prevent sharing of data in public repositories. According to a contract signed with the NPR and the SSB, the project is not allowed to forward data, or subsets of data, to other researchers, except project members named in the Data Protection Impact Assessment (DPIA). Furthermore, we are required to delete the linked dataset by 31 December 2023. Researchers with approval of an exemption from professional secrecy requirements for the use of personal health data in research from the 
Regional Committee for Medical and Health Research Ethics (REK) would be able to create an almost identical (updated) dataset by applying to the NPR and the SSB.

Supplemental material This content has been supplied by the author(s). It has not been vetted by BMJ Publishing Group Limited (BMJ) and may not have been peer-reviewed. Any opinions or recommendations discussed are solely those of the author(s) and are not endorsed by BMJ. BMJ disclaims all liability and responsibility arising from any reliance placed on the content. Where the content includes any translated material, BMJ does not warrant the accuracy and reliability of the translations (including but not limited to local regulations, clinical guidelines, terminology, drug names and drug dosages), and is not responsible for any error and/or omissions arising from translation and adaptation or otherwise.

Open access This is an open access article distributed in accordance with the Creative Commons Attribution Non Commercial (CC BY-NC 4.0) license, which permits others to distribute, remix, adapt, build upon this work non-commercially, and license their derivative works on different terms, provided the original work is properly cited, appropriate credit is given, any changes made indicated, and the use is non-commercial. See: http://creativecommons.org/licenses/by-nc/4.0/.

ORCID iD

Frank Olsen http://orcid.org/0000-0001-5392-2736

\section{REFERENCES}

1 Glover JA. The incidence of tonsillectomy in school children. Proc $R$ Soc Med 1938;31:1219-36.

2 Wennberg J, Gittelsohn A. Small area variations in health care delivery: a population-based health information system can guide planning and regulatory decision-making. Science 1973;182:1102-8.

3 Perrin JM, Homer CJ, Berwick DM, et al. Variations in rates of hospitalization of children in three urban communities. N Engl J Med 1989;320:1183-7.

4 Arora S, Cheung CR, Sherlaw-Johnson C, et al. Use of age-specific Hospital catchment populations to investigate geographical variation in inpatient admissions for children and young people in England: retrospective, cross-sectional study. BMJ Open 2018;8:e022339.

5 Moen A, Olsen F, Uleberg B. Child healthcare atlas for Norway. English version June 2017, ISBN: 978-82-93141-27-3, 2015.

6 Wennberg JE. Tracking Medicine: A researcher's quest to understand health care. New York: Oxford University Press, 2011.

7 Kaarbøe O, Carlsen F. Socioeconomic status and children's need for hospital services. Implications for need-based resource allocation models. Nordic Journal of Health Economics 2017;5:54-69.

8 Petrou S, Kupek E. Socioeconomic differences in childhood Hospital inpatient service utilisation and costs: prospective cohort study. J Epidemiol Community Health 2005;59:591-7.

9 Verlinde E, De Laender N, De Maesschalck S, et al. The social gradient in doctor-patient communication. Int $J$ Equity Health 2012;11:12.

10 Arpey NC, Gaglioti AH, Rosenbaum ME. How socioeconomic status affects patient perceptions of health care: a qualitative study. J Prim Care Community Health 2017;8:169-75.

11 Bernheim SM, Ross JS, Krumholz HM, et al. Influence of patients' socioeconomic status on clinical management decisions: a qualitative study. Ann Fam Med 2008;6:53-9.

12 WHO. The Global Health Observatory. SDG Target 3.2. Newborn and child mortality. Under-five deaths. Child mortality levels: Probability of dying per 1000 live births. Child mortality - Probability of dying per 1000 live births. Available: https://www.who.int/data/gho/ data/indicators/indicator-details/GHO/under-five-mortality-rate-( probability-of-dying-by-age-5-per-1000-live-births) [Accessed Jan 2020].

13 Moen A, Rønnestad A, Stensvold HJ. The Norwegian neonatal healthcare atlas. English version June 2017, ISBN: 978-82-93141-28$0,2016$.
14 Fisher ES, Wennberg JE, Stukel TA, et al. Associations among hospital capacity, utilization, and mortality of US Medicare beneficiaries, controlling for sociodemographic factors. Health Serv Res 2000;34:1351-62.

15 Allison P. Survival analysis using SAS. A practical guide. 2nd Edition. Cary, NC: SAS Institute Inc, 2010.

16 Halldórsson M, Kunst AE, Köhler L, et al. Socioeconomic differences in children's use of physician services in the Nordic countries. $J$ Epidemiol Community Health 2002;56:200-4.

17 Gong T, Lundholm C, Rejnö G, et al. Parental socioeconomic status, childhood asthma and medication use--a population-based study. PLoS One 2014;9:e106579.

18 Filc D, Davidovich N, Novack L, et al. Is socioeconomic status associated with utilization of health care services in a single-payer universal health care system? Int J Equity Health 2014;13:115.

19 Michel M, Alberti C, Carel J-C, et al. Association of pediatric inpatient socioeconomic status with hospital efficiency and financial balance. JAMA Netw Open 2019;2:e1913656.

20 Elofsson S, Undén AL, Krakau I. Patient charges--a hindrance to financially and psychosocially disadvantage groups seeking care. Soc Sci Med 1998;46:1375-80.

21 Kachmar AG, Connolly CA, Wolf S, et al. Socioeconomic status in pediatric health research: a scoping review. J Pediatr 2019;213:163-70.

22 Tsenkova V, Pudrovska T, Karlamangla A. Childhood socioeconomic disadvantage and prediabetes and diabetes in later life: a study of biopsychosocial pathways. Psychosom Med 2014;76:622-8.

23 Gehring U, Pattenden S, Slachtova $\mathrm{H}$, et al. Parental education and children's respiratory and allergic symptoms in the pollution and the young (PATY) study. Eur Respir J 2006;27:95-107.

24 Heinrich J, Popescu MA, Wjst M, et al. Atopy in children and parental social class. Am J Public Health 1998;88:1319-24.

25 du Prel X, Krämer U, Behrendt H, et al. Preschool children's health and its association with parental education and individual living conditions in East and West Germany. BMC Public Health 2006;6:312.

26 Mielck A, Reitmeir P, Wjst M. Severity of childhood asthma by socioeconomic status. Int J Epidemiol 1996;25:388-93.

27 Erdmann F, Hvidtfeldt UA, Sørensen M, et al. Socioeconomic differences in the risk of childhood central nervous system tumors in Denmark: a nationwide register-based case-control study. Cancer Causes Control 2020;31:915-29.

28 Finnvold JE. Access to specialized health care for asthmatic children in Norway: the significance of parents' educational background and social network. Soc Sci Med 2006;63:1316-27.

29 Jansen T, Rademakers J, Waverijn G, et al. The role of health literacy in explaining the association between educational attainment and the use of out-of-hours primary care services in chronically ill people: a survey study. BMC Health Serv Res 2018;18:394.

30 Friis K, Lasgaard M, Rowlands G, et al. Health literacy mediates the relationship between educational attainment and health behavior: a Danish population-based study. J Health Commun 2016;21:54-60.

31 Baker DW, Gazmararian JA, Williams MV, et al. Functional health literacy and the risk of hospital admission among Medicare managed care enrollees. Am J Public Health 2002;92:1278-83.

32 Tak HJ, Ruhnke GW, Meltzer DO. Association of patient preferences for participation in decision making with length of stay and costs among hospitalized patients. JAMA Intern Med 2013;173:1195-205.

33 Salvador Ágata, Crespo C, Roberto MS, et al. Do parents of children with cancer want to participate in treatment decision-making? Support Care Cancer 2020;28:1059-67.

34 Goodman DC. Unwarranted variation in pediatric medical care. Pediatr Clin North Am 2009;56:745-55.

35 Cutler D, Skinner JS, Stern AD, et al. Physician beliefs and patient preferences: a new look at regional variation in health care Spendingf. Am Econ J Econ Policy 2019;11:192-221. 